\title{
Aproximación numérica de Vega bajo el modelo de volatilidad estocástica de Heston, utilizando el Método de Trayectorias-Euler
}

\author{
Ana María Serrato Polanía*
}

* Magíster en Finanzas, Universidad Externado de Colombia. anamaserrato@gmail.com Fecha de recepción: 16 de febrero de 2015

Fecha de aceptación: 01 de abril de 2015.

Forma de citar:

Serrato Polanía,A. M. (2015). Aproximación numérica de Vega bajo el modelo de volatilidad estocástica de Heston, utilizando el Método de Trayectorias - Euler. ODEON, 9, pp. 81-112. DOI: http://dx.doi.org/10.18601/17941113.n9.03 



\section{Introducción}

El modelo de mercado de Black-Scholes es conocido como el pilar de las finanzas cuantitativas modernas, cuya fórmula cerrada trabaja bajo supuestos específicos, entre ellos volatilidad constante como supuesto fundamental, simplificando el desarrollo de la ecuación; esto nos hace pensar que el mercado es completo, que los riesgos pueden ser cubiertos por el activo subyacente y que no debe estimarse una prima por riesgo de volatilidad.

Sin embargo, en el mercado real se observa que la volatilidad fluctúa (es estocástica), esto implica que al valorar opciones con Black-Scholes se presentan altos niveles de error en la volatilidad que ejercen presión en el sistema económico y financiero (lo cual permite observar el grado de riesgo que percibe el mercado), afectando el precio de las opciones y el desempeño del mercado en general, lo que a su vez puede representar pérdidas significativas de dinero.

Entonces, ¿qué pasa cuando la volatilidad no es constante y estamos valorando opciones? Una de las opciones es migrar a un modelo donde la volatilidad sea estocástica.

En vista de lo anterior, se han desarrollado diversos modelos que tratan de capturar las características observadas en los mercados financieros, en donde se deja de lado el supuesto de volatilidad constante para trabajar con volatilidad estocástica, es decir, la volatilidad de la volatilidad. Algunos de ellos son la volatilidad como función del tiempo, modelos econométricos (Garch, Dtarch), modelos de difusión con saltos y el modelo de volatilidad estocástica desarrollado por Heston (SV por sus siglas en inglés), bajo el cual se desarrollará este trabajo.

Sin embargo, a pesar de que los modelos mencionados representan el comportamiento de las series que tienen volatilidad no constante, los modelos de valoración de opciones no encuentran solución analítica de las griegas, especialmente de Vega (sensibilidad en el valor de una opción debido a cambios en la volatilidad) en donde se enfoca nuestro caso de estudio.

Para esto se propone discretizar las ecuaciones diferenciales resultantes del modelo de Heston con el fin de aproximar las soluciones usando el Método de Discretización de Euler Maruyama para obtener la trayectoria de $S_{T}$. Posteriormente, se implementará el Método de Trayectorias desarrollado en conjunto con el Método de Diferencias Finitas, para hacer la aproximación de las griegas, con el fin de establecer un mecanismo que permita considerar la sensibilidad de las opciones a la volatilidad en modelos de gestión de riesgo. Esto permitiría cubrir la exposición en el mercado, y a su vez limitar las pérdidas y la incertidumbre que provoca el mercado de divisas, cuyas fluctuaciones suponen un impacto directo en 
los inversionistas o administradores de portafolios, ya que si la volatilidad cambia, es de interés conocer el efecto de estos cambios en el portafolio de activos.

\section{El modelo de Black-Scholes y su supuesto de volatilidad constante}

El modelo de mercado de Black-Scholes (1973) y Merton (1973) se basa en el supuesto de que el precio del activo subyacente sigue un proceso de difusión lognormal con volatilidad constante.

El modelo Black-Scholes involucra dos activos, un activo riesgoso cuyo precio evoluciona desde un valor inicial de acuerdo con la siguiente expresión:

$$
d S=\mu S d t+\sigma S d z
$$

El otro activo es un bono o activo libre de riesgo $\Delta \Pi=r \prod \Delta t$ que evoluciona en forma determinística.

$$
\Delta f=\left(\frac{\partial f}{\partial S} \mu S+\frac{\partial f}{\partial t}+\frac{1}{2} \frac{\partial^{2} f}{\partial S^{2}} \sigma^{2} S^{2}\right) \Delta t+\frac{\partial f}{\partial S} \sigma S \Delta z
$$

Donde:

$\Delta S$ y $\Delta f$ cambios en $f$ y $S$ en un pequeño intervalo de tiempo $\Delta t$.

Teniendo en cuenta lo anterior, se construye un portafolio apropiado de la siguiente manera:

$-1:$ derivado +

$\frac{\partial f}{\partial S}:$ Acciones

Al sustituir $\Delta f$ y $\Delta S$ se obtiene la ecuación diferencial de Black-Scholes y Merton, dada por:

$$
\frac{\partial f}{\partial t}+r S \frac{\partial f}{\partial S}+\frac{1}{2} \sigma^{2} S^{2} \frac{\partial^{2} f}{\partial S^{2}}=r f
$$


En 1973, Fisher Black y Myron Scholes consideran, bajo este marco, el problema de valorar una opción de compra europea con precio de ejercicio $K$ y maduración $T$, donde el activo subyacente de la opción sigue una medida de probabilidad bajo un proceso de Wiener, es decir, una probabilidad riesgo neutral. Black y Scholes muestran que es posible replicar esta opción, cuyo valor al ejercicio está dado por la expresión $(S(T)-K)^{+}$y que, por tanto, puede ser valorada por no arbitraje.

A partir de lo anterior, se tiene que el valor de una opción call europea, en el momento en que es pactada $(c)$, está determinado por:

$$
c=S N(d 1)-e^{-r T} K N(d 2)
$$

Donde:

$\mathrm{N}(\cdot)$ : distribución acumulada de la normal estándar y las variables $d 1$ y $d 2$ están definidas por (Moreno Trujillo, 2012):

$$
d 1=\frac{\ln (S / K)+\left(r+1 / 2 \sigma^{2}\right) T}{\sigma \sqrt{T}} ; d 2=d 1-\sigma \sqrt{\mathrm{T}}
$$

Una de las ventajas que brinda el modelo del marco de valoración de opciones de Black-Scholes es la posibilidad de dividir el problema de cobertura o replicación de derivados en términos de factores de riesgo. Dichos factores, que en adelante llamaremos griegas, son los siguientes (tabla 1):

Tabla 1. Descripción de las griegas

\begin{tabular}{|l|l|}
\hline \multicolumn{2}{|c|}{ Griegas } \\
\hline Delta $(\Delta)$ & Cambios en el valor de una opción debido a cambios en el activo subyacente $S, \frac{\partial v}{\partial s}$. \\
\hline Gamma $(\Gamma)$ & Cambios en el valor de Delta $(\Delta)$ de una opción, $\frac{\partial v^{2}}{\partial^{2} s}$. \\
\hline Theta $(\Theta)$ & Cambios en el valor de una opción debido a cambios en el plazo $(T-t), \frac{\partial v}{\partial t}$. \\
\hline Vega $(v)$ & Cambios en el valor de una opción debido a cambios en la volatilidad $\sigma, \frac{\partial v}{\partial \sigma}$. \\
\hline Rho $(\varrho)$ & Cambios en el valor de una opción debido a cambios en la volatilidad $\sigma, \frac{\partial v}{\partial \sigma}$. \\
\hline
\end{tabular}




\subsection{Alternativas a Black-Scholes y modelos de volatilidad estocástica}

Modelos estadísticos. Los métodos de estimación que asume un modelo estadístico no realizan una estimación directa de la volatilidad. Estos métodos modelizan tanto la serie de rentabilidades como la varianza de la rentabilidad, y, a partir de esta última, definen la desviación típica como volatilidad; el ajuste del modelo para la varianza permitirá obtener la estimación de la volatilidad. Se destacan entre ellos los modelos Garch, Dtarch y los modelos de volatilidad estocástica.

Los modelos Garch pueden producir conglomerados de alta volatilidad. El modelo estima dos ecuaciones simultáneas que explican la evolución de la rentabilidad en función de rentabilidades pasadas, modelando la evolución de la varianza de la rentabilidad, realizando así la estimación de la volatilidad. Sin embargo, este modelo tiene limitaciones en la predicción ya que para obtener una estimación robusta necesita una cantidad considerable de datos y el modelo no está diseñado para generar predicciones de la varianza a varios pasos.

Modelos de volatilidad estocástica. El modelo de volatilidad estocástica SV, introducido por Taylor en 1986, y por Shephard en el mismo año, en donde se supone que la rentabilidad depende de una variable no observable (variable latente), que sigue un proceso estocástico ARMA, y de una variable aleatoria independiente e idénticamente distribuida (Medina, 2010).

Los modelos de SV especifican el algoritmo de la volatilidad como un proceso estocástico lineal, en donde la volatilidad se considera un componente no observable de la serie modelada a través de un proceso lineal autorregresivo.

Los autores Hull y White (1987), Melino y Turnbull (1990), Wiggins (1987) entre otros, generalizan la tradicional especificación del movimiento browniano geométrico, sugiriendo un proceso propio para la volatilidad estocástica. Así Heston (1993), en una contribución clave para la literatura de valoración de opciones admite, a diferencia de los anteriores, la correlación entre los brownianos del subyacente y la varianza, además de obtener expresiones de valoración de opciones mediante la inversión de Fourier de la función característica condicional (González, 2008).

De otro lado, el modelo de Heston (1993) es considerado uno de los más representativos, ya que el proceso para la volatilidad es no negativo y presenta reversión a la media (lo cual se observa en los mercados) y tiene una solución en forma cerada para opciones plain vanilla. También permite explicar el smile de volatilidad. 


\section{El modelo de Heston (1993)}

El modelo de Heston es uno de los más utilizados en modelos de volatilidad estocástica, su atractivo recae en que es uno de los modelos más robustos.

El modelo de Heston propone lo siguiente:

$$
\begin{gathered}
d S t=\mu S t d t+\sqrt{V} S_{t} d W_{t}^{1} \\
d V t=\kappa(\theta-V t) d t+\eta \sqrt{V_{t}} d W_{t}^{2} \\
d W t^{1} d W t^{2}=\rho d t
\end{gathered}
$$

Donde:

$\{S t\} t \geq 0$ y $\{V t\} t \geq 0$ : procesos de precio y volatilidad.

$\left\{W t^{1}\right\} t \geq 0,\left\{W t^{2}\right\} t \geq 0$ : procesos de movimiento browniano correlacionados (con parámetro de correlación $\rho)$.

$\{V t\} t \geq 0$ : raíz cuadrada del proceso de reversión a la media, primero usado por Cox, Ingersoll, y Ross (1985), con media a largo plazo $\theta$ y una tasa de reversión $\kappa, \eta$ : volatilidad de la volatilidad.

Todos los parámetros $\mu, \kappa, \theta, \eta$, $\rho$, están en tiempo y estado homogéneos, donde $\rho$ puede ser interpretado como la correlación entre los retornos lognormales y la volatilidad del activo. Intuitivamente, si $\rho>0$, entonces la volatilidad aumentará a medida que el precio/retorno del activo aumente; inversamente, si $\rho>0$ la volatilidad aumentará cuando el precio/retorno del activo disminuya. $\eta$ afecta la curtosis de la distribución. Cuando $\eta$ es 0 , la volatilidad es determinística $\mathrm{y}$, por tanto, los retornos lognormales serán normalmente distribuidos. $\kappa$ es el parámetro de reversión a la media, que puede ser interpretado como una representación del grado del clúster de volatilidad, los cuales son observados en el mercado, es decir, que grandes variaciones de precios tienen más probabilidades de ser seguidas por grandes variaciones de precios.

Las características del modelo de Heston antes mencionadas posibilitan una gran cantidad de distribuciones. Esto hace que el modelo sea robusto y, por tanto, aborda las deficiencias del modelo Black-Scholes-Merton, ofreciendo un marco para valorar una variedad de opciones que están más cerca de la realidad (Moodley, 2005). 
Sin embargo, cuando se trata de encontrar precios o valores de las griegas el primer problema que se encuentra es que, bajo el modelo de Heston, el precio del mercado del riesgo de la volatilidad es difícil de estimar ya que no otorga una solución exacta que pueda ser calculada directamente.

\subsection{Métodos para aproximar las griegas, entre ellas Vega}

\subsubsection{Discretización de Euler}

Consideramos un proceso $S$ que satisface una ecuación diferencial parcial de la forma:

$$
\begin{gathered}
d S(t)=a(S(t)) d t+b(S(t)) d W(t) \\
S(0)=0
\end{gathered}
$$

Donde:

$S$ toma valores en $\mathbb{R}^{d}$

$W$ es un movimiento browniano estándar $m$-dimensional en cuyo caso $a$ toma valores en $\mathbb{R}^{d}$ y $b$ toma valores en $\mathbb{R}^{d \times m}$.

Usamos $\hat{S}$ para denotar una aproximación a $S$ en tiempo discreto. La aproximación de Euler en una cuadrícula de tiempo $0=t_{0}<t_{1}<\cdots<t_{m}=T$, está definida por:

$$
\hat{S}\left(t_{i+1}\right)=\hat{S}\left(t_{i}\right)+a\left(\hat{S}\left(t_{i}\right)\right)\left[t_{i+1}-t_{i}\right]+b\left(\hat{S}\left(t_{i}\right)\right) \sqrt{t_{i+1}-t} Z_{i+1}
$$

Donde:

$$
\hat{S}(0)=S(0) ; i=0, \ldots, m-1
$$

$Z_{1}, Z_{2}, \ldots, Z_{m}$ son normales estándar independientes.

Para suavizar la notación, fijamos la atención sobre una cuadrícula con un espaciado $h$ fijo, lo que significa que $t_{i}=i h$.

Con intervalos de tiempo fijos $h>0$, podemos escribir $\hat{S}(i h)$ como $\hat{S}(i)$ y escribir el esquema de Euler como (Glasserman, 2003): 


$$
\hat{S}(i+1)=\hat{S}(i)+a(\hat{S}(i)) h+b(\hat{S}(i)) \sqrt{h} Z_{i+1}
$$

Así como la discretización de Euler funciona para una ecuación, también puede ser usada para un sistema de ecuaciones estocásticas, lo que quiere decir que las ecuaciones diferenciales de Heston (ver sección 2) que expresadas matricialmente:

$$
d\left[\begin{array}{l}
S_{t} \\
V_{t}
\end{array}\right]=\left[\begin{array}{c}
\mu S_{t} \\
\kappa(\theta-V t)
\end{array}\right] d t+\left[\begin{array}{cc}
V_{t} S & 0 \\
0 & \eta \sqrt{V_{t}}
\end{array}\right]\left[\begin{array}{l}
d W_{t}^{1} \\
d W_{t}^{2}
\end{array}\right]
$$

pueden discretizarse bajo el esquema de Euler, para obtener:

$$
\begin{aligned}
& \hat{S}(i+1)=\hat{S}(i)+\mu \hat{S}(i) h+\hat{V}(i) \hat{S}(i) \sqrt{h} Z_{i+1}^{1} \\
& \hat{V}(i+1)=\kappa(\theta-\hat{V}(i)) h+\eta \sqrt{\hat{V}(i)} \sqrt{h} Z_{i+1}^{2}
\end{aligned}
$$

\subsection{Método de Trayectorias-Euler}

Este método nos permite realizar aproximaciones numéricas de las griegas en los modelos de mercados financieros.

En forma general, el precio de un derivado europeo se puede ver como $\alpha(\theta)=$ $\mathrm{E}[f(\theta)]$, donde $f(\theta)$ es la función de pagos descontada del derivado, en función de unos parámetros definidos como $\theta$.

Recordemos que bajo ciertas condiciones de regularidad la derivada de una esperanza es igual a la esperanza de la derivada, así:

$$
\alpha^{\prime}(\theta)=\frac{d E[f(\theta)]}{d \theta}=E\left[\frac{d f(\theta)}{d \theta}\right]
$$

Por tanto, las griegas se pueden expresar como la ecuación 13, dependiendo de qué parámetro se tome como theta. En este punto es preciso aclarar que el valor esperado resultante de la ecuación 16 es neutral al riesgo.

Ya que este trabajo tiene como objetivo la aproximación de $\mathrm{Vega}^{1}$, entonces, el parámetro que nos interesa es $\sigma_{0} \mathrm{y}$, por ende, el valor esperado de interés es:

1 Vega se debe interpretar de la siguiente manera: por ejemplo, si Vega es igual a 30, entonces se divide 30 entre $100(30 / 100)$ que es igual a 0,30 centavos (porque se divide entre 100), este será el cambio en el precio de la opción ante un cambio de $1 \%$ en la volatilidad. 


$$
E\left[\frac{d f\left(S_{T}, V_{o}, T, r\right)}{d \sigma_{0}}\right]=E\left[\left(\frac{d f}{d S_{T}}\right)\left(\frac{d S_{T}}{d V_{0}}\right)\left(\frac{d V_{0}}{d \sigma_{0}}\right)\right]
$$

Donde:

$\left(\frac{d f}{d S_{T}}\right)$ derivada del precio de la opción respecto al precio del activo al vencimiento. $\left(\frac{d S_{T}}{d V_{0}}\right)$ derivada del precio del activo al vencimiento respecto a la varianza inicial. $\left(\frac{d V_{0}}{d \sigma_{0}}\right)$ derivada de la varianza inicial respecto al sigma inicial.

Teniendo en cuenta lo anterior, el método de Trayectorias - Euler propone aproximar este valor esperado utilizando discretización de Euler y Monte Carlo para crear trayectorias de $S_{T}$, y diferencias finitas para evaluar $\frac{S_{T}}{V_{0}}$.

Específicamente, el cálculo de Vega se hará para un call europeo Plain Vanilla, del cual se conoce que:

$$
\frac{d f}{d S_{T}}=e^{-r T} 1_{\left(S_{T}>K\right)}^{2}
$$

Reemplazando en la ecuación 17 tenemos que:

$$
E\left[e^{-r T} 1_{\left(S_{T}>K\right)}\left(\frac{d S_{T}}{d V_{0}}\right)\left(\frac{d V_{0}}{d \sigma_{0}}\right)\right]
$$

donde $e^{-r T}$ y $S_{0}$ son constantes. Como se mencionó, $S_{T}$ y $1_{\left(S_{T}>K\right)}$ se calcularán utilizando discretización de Euler y simulación de Monte Carlo.

A continuación, para realizar la aproximación de $\frac{d S_{T}}{d V_{0}}$ se implementará el Método de Diferencias Finitas ${ }^{3}$, el cual parte de:

$2 \quad$ En donde $\frac{d}{d x}(x-K)^{+}=\left\{\begin{array}{l}0 \text { si } x<K \\ 1 \text { si } x>K\end{array}=1_{\{x>K\}}\right\}$

3 Método basado en aproximaciones que permiten reemplazar ecuaciones diferenciales por ecuaciones de diferencia; dichas expresiones son de forma algebraica y relacionan el valor de la variable dependiente, en un punto dentro de la región de solución, con sus valores en algunos puntos vecinos. 


$$
\frac{d f(x)}{d x}=\lim _{h \rightarrow \infty} \frac{f(x+h)-f(x)}{h}
$$

o con aproximación central de la derivada

$\frac{d f(x)}{d x} \cong \frac{f(x+1 / 2 h)-f(x-1 / 2 h)}{h}$
Reemplazando $\frac{d S_{T}}{d V_{0}}$ por su aproximación central en la ecuación 18 , tenemos que

$$
E\left[\left(\frac{d f}{d S_{T}}\right)\left(\frac{S_{T}\left(V_{0}+1 / 2 h\right)-S_{T}\left(V_{0}-1 / 2 h\right)}{h}\right)\left(\frac{d V_{0}}{d \sigma_{0}}\right)\right]
$$

Donde:

$h$ : variación de la varianza inicial.

Nuevamente se puede usar el método de Trayectorias - Euler para evaluar ese segundo término del valor esperado.

Y, por último, $\frac{d V_{0}}{d \sigma_{0}}$ es una constante y equivale a $2 \sigma_{0}$, así:

$$
E\left[\left(\frac{d f}{d S_{T}}\right)\left(\frac{d S_{T}}{d V_{0}}\right) 2 \sigma_{0}\right]
$$

Finalmente, reemplazando todos los términos en la ecuación 17, tenemos que:

$$
E\left[e^{-r T} 1_{\left(S_{T}>K\right)}\left(\frac{S_{T}\left(V_{0}+1 / 2 h\right)-S_{T}\left(V_{0}-1 / 2 h\right)}{h}\right) 2 \sigma_{0}\right]
$$

Debe tenerse en cuenta que:

a. Se simulan dos caminos con los mismos aleatorios y dos vectores diferentes de $V_{0}$. b. Se aplica la aproximación central.

c. Hay que recordar que cada trayectoria simulada también se usa para hallar el valor de la función indicadora. 
En otras palabras, para aproximar $\frac{d S_{T}}{d V_{0}}$ tomamos el mismo set de aleatorios que utilizamos anteriormente, pero le cambiamos $V_{0}$ (para los dos casos: positivo y negativo), teniendo en cuenta que en el modelo de Black-Scholes $V_{0}$ es constante, y en el modelo de Heston $V_{0}$ es el valor inicial de la varianza que es controlado por $\kappa, \theta$ y $\eta$ (según la ecuación 7). Este proceso se realiza con el fin de revisar el comportamiento de las trayectorias cuando la volatilidad aumenta o disminuye, y se repite el proceso tantas veces como sea necesario.

Como resultado, obtenemos dos trayectorias perturbadas por el cambio en la volatilidad inicial, y así con el valor de la perturbación en sigma se puede aproximar la derivada.

\subsection{Resumen del algoritmo}

A continuación se muestra el algoritmo propuesto para aproximar de Vega, implementando el Método de Trayectorias Euler, utilizando Discretización de Euler, Monte Carlo y Diferencias Finitas, el cual está generalizado para variables.

Por medio de este algoritmo se genera un set de aleatorios correlacionados y se discretiza el sistema de ecuaciones que representan el comportamiento del activo riesgoso, lo cual permite generar trayectorias que posteriormente serán perturbadas permitiendo así calcular realizaciones de $\left[\frac{d f(\theta)}{d \theta}\right]$; finalmente, se toma el promedio de los valores generados para así obtener la aproximación de Vega. 


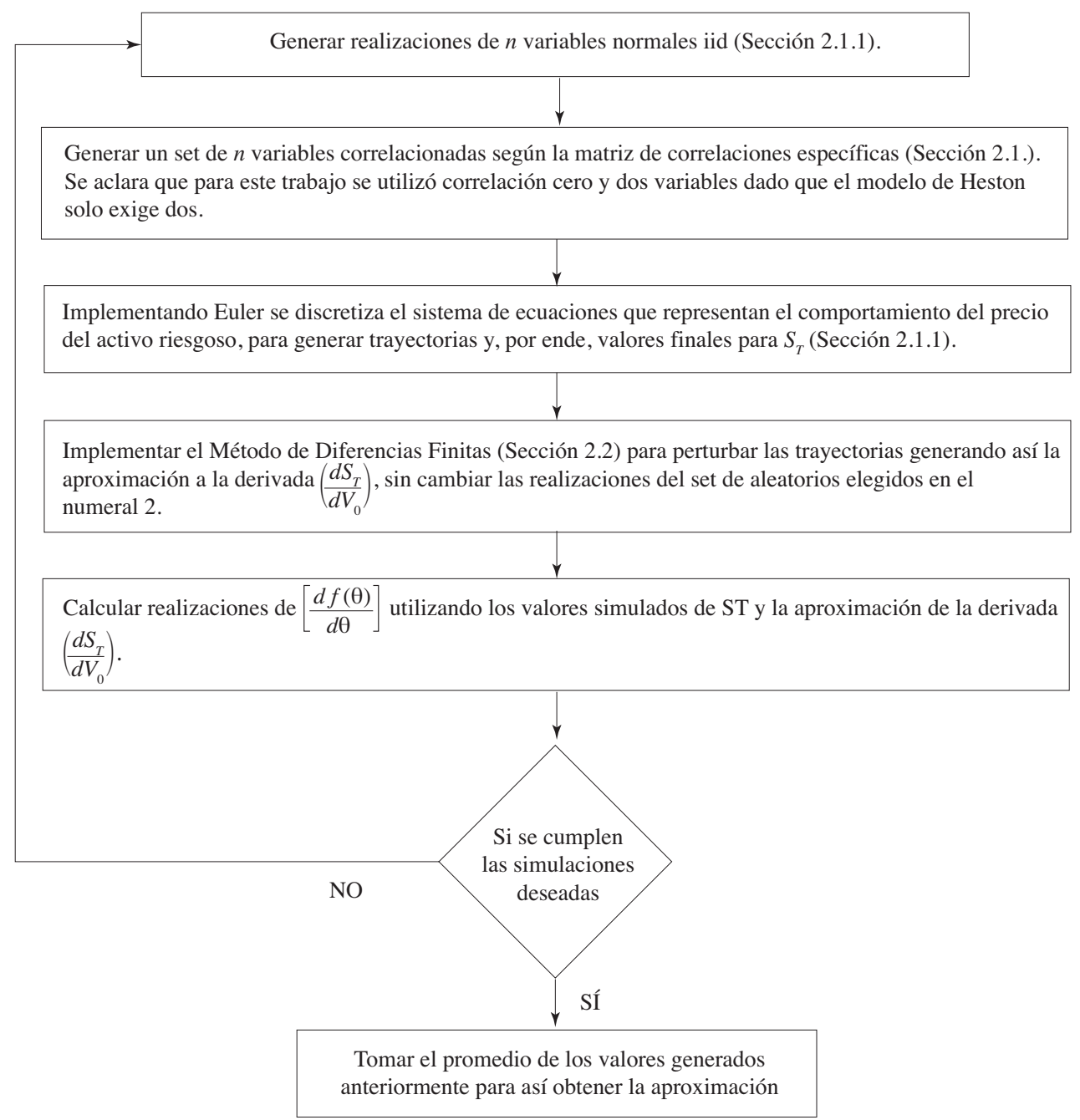

\section{Resultados}

El experimento consiste en mostrar la efectividad del Método de TrayectoriasEuler, evaluando $\mathrm{Vega}^{4}$ en el modelo de Black-Scholes, y comparando el valor real de Vega obtenido con la fórmula cerrada de Black-Scholes con la aproxima-

$4 \quad$ Vega se debe interpretar de la siguiente manera: por ejemplo, si Vega es igual a 30, entonces se divide 30 entre 100 (30/100) que es igual a 0,30 centavos (porque se divide entre 100), este será el cambio en el precio de la opción ante un cambio de $1 \%$ en la volatilidad. 
ción numérica de Vega bajo el Método de Trayectorias-Euler. Posteriormente, se aproxima Vega en el modelo de Heston (1993) con el Método de Trayectorias-Euler.

\subsection{Comparación de Vega obtenido por el método de Trayectorias- Euler vs. Vega Black-Scholes analítico}

El Vega de una opción está definido como ${ }^{v}=\frac{d f}{d \sigma_{0}}$, donde $d f$ es el precio de la opción $\sigma_{0}$ y es la volatilidad del precio del activo inicial. Luego:

$v=\mathrm{S}_{0} \sqrt{\mathrm{T}} \Phi(d 1)^{5}$

Se procede a probar el método numérico propuesto para aproximar Vega en BlackScholes, ejecutándolo en 16 escenarios diferentes con un intervalo de confianza del $99 \%$, parámetros fijos: $S 0=100, R_{f}=1 \%, T=1, m=\{1,4,6,12,52\}$ y parámetros variables: $K=80: 05: 120$ y $\sigma=\{0,05 ; 0,1 ; 0,3 ; 0,5\}$. En este trabajo se presentarán cinco casos representativos, que arrojan como resultado un Vega de Black-Scholes determinístico como se muestra en la tabla 2:

Tabla 2. Cálculo del Vega analítico en el Modelo de Black-Scholes

\begin{tabular}{|c|c|c|c|c|c|}
\hline Parámetro & Caso base & Caso 1 & Caso 2 & Caso 3 & Caso 4 \\
\hline $\mathrm{K}$ & 105 & 90 & 105 & 105 & 120 \\
\hline$\sigma$ & 0,3 & 0,3 & 0,1 & 0,5 & 0,3 \\
\hline v analítico & 39,8857 & 34,5832 & 37,6805 & 39,3056 & 36,4584 \\
\hline
\end{tabular}

Posteriormente, se aplica el Método de Trayectorias-Euler, tomando como caso base $(K=105, \sigma=0,3)$ y los mismos valores iniciales de: $S 0=100, R_{f}=1 \%, T=1$, con valores de $m=\{1,4,6,12,52\}$ para 1.000 y 100.000 simulaciones, como se observa en la tabla 3 .

5 Para ver la demostración de esta derivación, remítase al documento original "Aproximación numérica de Vega bajo el modelo de volatilidad estocástica de Heston, utilizando el Método de Trayectorias-Euler". 
Tabla 3. Vega aproximado con el Método de Trayectorias-Euler. Parámetros:

$S 0=100, R_{f}=1 \%, T=1, m=\{1,4,6,12,52\}, K=105, \sigma=0,3$, Intervalo de Confianza $=99 \%$

\begin{tabular}{|c|r|r|r|r|r|r|r|}
\hline \multirow{2}{*}{$\mathbf{N}$} & Iteraciones & $\mathbf{v}$ Analítico & $\begin{array}{c}\text { Error de } \\
\text { estimación }\end{array}$ & $\begin{array}{c}\mathbf{v} \text { Aproxi- } \\
\text { mado }\end{array}$ & $\begin{array}{c}\text { Tiempo } \\
\text { transcurrido } \\
\text { (segundos) }\end{array}$ & $\begin{array}{c}\text { Intervalo } \\
\text { superior }\end{array}$ & $\begin{array}{c}\text { Intervalo } \\
\text { inferior }\end{array}$ \\
\hline \multirow{2}{*}{1} & 1000 & 39.8857 & 1.8040 & 39.3630 & 0.1302 & 42.971 & 35.755 \\
\cline { 2 - 8 } & 100000 & 39.8857 & 0.1838 & 39.1470 & 12.1331 & 39.5146 & 38.7794 \\
\hline \multirow{2}{*}{4} & 1000 & 39.8857 & 2.3164 & 36.9905 & 0.4241 & 41.6233 & 32.3577 \\
\cline { 2 - 9 } & 100000 & 39.8857 & 0.2432 & 40.648 & 41.7979 & 41.1344 & 40.1616 \\
\hline \multirow{2}{*}{6} & 1000 & 39.8857 & 2.3046 & 36.3111 & 0.6175 & 40.9203 & 31.7019 \\
\cline { 2 - 9 } & 100000 & 39.8857 & 0.2492 & 40.3635 & 61.3388 & 40.8619 & 39.8651 \\
\hline \multirow{2}{*}{12} & 1000 & 39.8857 & 2.3938 & 38.1410 & 1.2041 & 42.9286 & 33.3534 \\
\cline { 2 - 9 } & 100000 & 39.8857 & 0.2578 & 40.1607 & 124.9468 & 40.6763 & 39.6451 \\
\hline \multirow{2}{*}{52} & 1000 & 39.8857 & 2.7006 & 39.2623 & 5.4569 & 44.6635 & 33.8611 \\
\cline { 2 - 8 } & 100000 & 39.8857 & 0.264 & 40.0815 & 558.8105 & 40.6095 & 39.5535 \\
\hline
\end{tabular}

Gráfico 1. Vega aproximado con el Método de Trayectorias-Euler. Parámetros: $S 0=100, R_{f}=1 \%, T=1, m=\{1,4,6,12,52\}, K=105, \sigma=0,3$, Intervalo de Confianza $=99 \%$.

Las estrellas simbolizan el valor analítico de Vega en el modelo de Black-Scholes

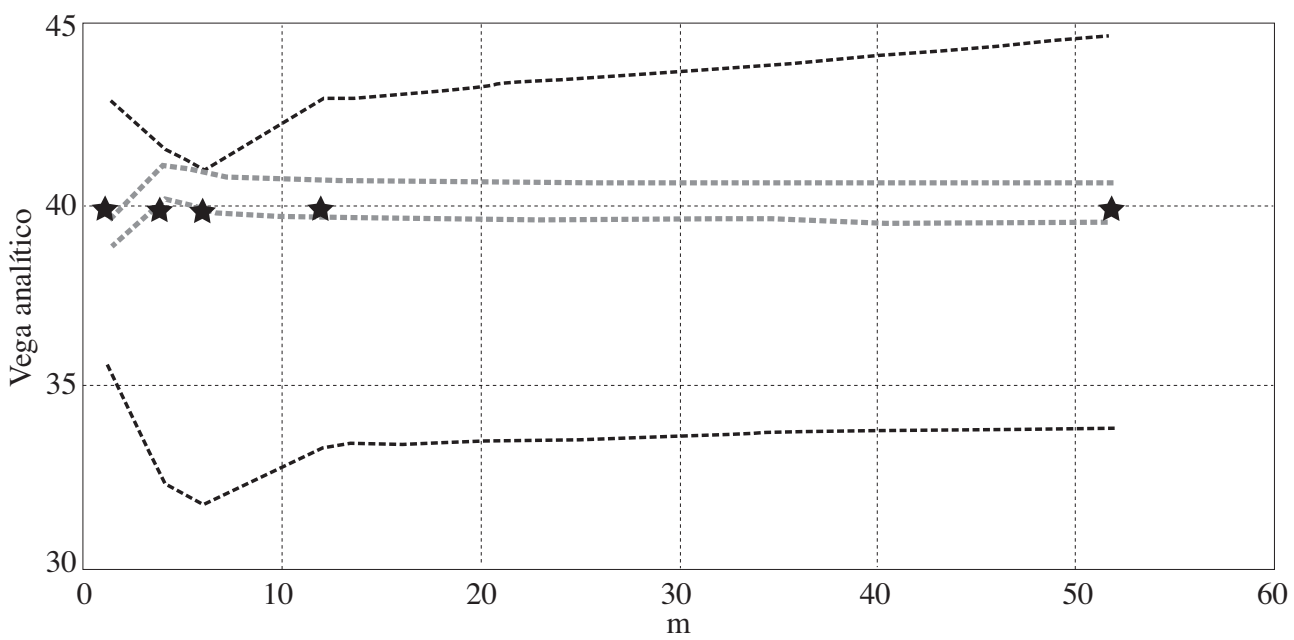


En el gráfico 1 se plasma el comportamiento del caso base con parámetros $K=105, \sigma=0,3, S 0=100, R_{f}=1 \%, T=1$, con valores de $m=\{1,4,6,12,52\}$; éste muestra que con 1.000 simulaciones el valor analítico del Vega de Black-Scholes con un intervalo de confianza del 99\%, es de 39,8857 (cambio de 0,398857 centavos en el precio de la opción ante un cambio de $1 \%$ en la volatilidad), y que cae dentro del intervalo construido; sin embargo, debido a que este número de simulaciones es pequeño hay una distancia de $-1,5878 \%$ entre el Vega analítico y el Vega aproximado, siendo este último de 39,2623 (0,392623 centavos) para $m=52$, por tal motivo, se procede a repetir el ejercicio con 100.000 simulaciones siguiendo el proceso del algoritmo que se muestra en la Sección 2.3.

Al realizar la prueba con 100.000 simulaciones en el caso base con parámetros $K=105, \sigma=0,3, S 0=100, R_{f}=1 \%, T=1$ con valores de $m=\{1,4,6,12,52\}$ se observa que la partición de $m$ cobra vital importancia puesto que para $m=\{1,4\}$ se presenta un sesgo hacia arriba del Vega aproximado respecto al Vega analítico; no obstante, para $m=\{52\}$ el Vega aproximado por el Método de Trayectorias-Euler está a una distancia de 0,4885\% respecto al Vega Analítico, lo que quiere decir que el método numérico que se está implementando es bueno.

En los casos en los que se variaron los parámetros $(K y \sigma)$, como se puede ver en la sección 5.1, se observa que el comportamiento es casi el mismo al descrito en la párrafo anterior, presentando sesgo en las primeras particiones; sin embargo, a medida que aumenta el $m$, el Vega analítico cae dentro del intervalo creado por simulación.

Es importante destacar que en el caso específico, con parámetros $K=120$, $\sigma=0,05$, no aparecen los intervalos para 1.000 simulaciones ya que en el experimento ningún valor del $S_{T}$ simulado superó el strike de 120 (ver sección 5.1).

En conclusión, se observa que para el caso base, con parámetros $K=105, \sigma=$ $0,3, S 0=100, R_{f}=1 \%, T=1$, al igual que para los demás casos desarrollados (ver tabla 2) con 1.000 y 100.000 simulaciones, el Vega analítico cayó dentro del intervalo creado por simulaciones con el Método de Trayectorias-Euler cuando $m \geq 12$.

El cuadro resumen que muestra los resultados del Vega Analítico vs. el Vega generado por el Método de Trayectorias-Euler bajo el modelo de Black-Scholes se puede observar en el anexo 9.

Dado que al realizar el experimento con el modelo de Black-Scholes se observa que el rango de estimación por el Método de Trayectorias-Euler abarca el valor del Vega analítico, se procede a implementar el método en el modelo de Heston (1993), en donde, como es de nuestro conocimiento, la volatilidad presenta un comportamiento estocástico y, además, el Vega no tiene solución cerrada. 


\subsection{Aproximación de Vega por el Método de Trayectorias - Euler bajo el modelo de volatilidad estocástica de Heston}

A diferencia del cálculo de Vega en el modelo de Black-Scholes, en donde se tiene el supuesto de volatilidad constante, en el modelo desarrollado por Heston (1993) la volatilidad es estocástica, y no tiene una fórmula analítica para el cálculo de Vega, por tanto, vamos a confiar en el intervalo de confianza de $99 \%$.

Para este experimento, se desarrollaron once casos con diferentes parámetros (el cuadro resumen de los resultados del Vega aproximado por el Método de Trayectorias-Euler bajo el modelo de Heston (1993) se puede consultar en el anexo 16. Los primeros cinco casos se realizan con las mismas variaciones para el strike y para el sigma del activo que se utilizaron en el Modelo de Black-Scholes (como se muestra en la tabla 4), con parámetros fijos: $S 0=100, R_{f}=1 \%, T=1$, con $m=\{1,4,6,12,52\}$, con un intervalo de confianza del $99 \%$.

Tabla 4. Parámetros iniciales para la aproximación de Vega en el Modelo de Heston

\begin{tabular}{|c|c|c|c|c|c|}
\hline Parámetro & Caso base & Caso 1 & Caso 2 & Caso 3 & Caso 4 \\
\hline $\mathrm{K}$ & 105 & 105 & 105 & 90 & 120 \\
\hline$\sigma$ & 0.3 & 0.15 & 0.5 & 0.3 & 0.3 \\
\hline$\kappa$ & 0.01 & 0.01 & 0.01 & 0.01 & 0.01 \\
\hline$\theta$ & 0.003 & 0.003 & 0.003 & 0.003 & 0.003 \\
\hline$\eta$ & 0.1 & 0.1 & 0.1 & 0.1 & 0.1 \\
\hline $\begin{array}{l}\text { V Método Trayecto- } \\
\text { rias - Euler (100.000 } \\
\text { sim y m = 12) }\end{array}$ & 7.3730 & 2.2657 & 8.0264 & 3.425 & 1.4722 \\
\hline
\end{tabular}

Teniendo en cuenta lo anterior, al realizar el experimento con 1.000 y 100.000 simulaciones, el caso base con parámetros fijos: $S 0=100, R_{f}=1 \%, T=1$, con $m=\{1,4,6,12,52\}$, y parámetros variables: $K=105, \sigma=0,3, \kappa=0,01, \theta=0,003$ y $\eta=0,1$, con un intervalo de confianza del $99 \%$, arroja los resultados que se observan en la tabla 5 . 
Tabla 5. Vega aproximado con el Método de Trayectorias-Euler. Parámetros: $S 0=100, R_{f}=1 \%, T=1, m=\{1,4,6,12,52\}, K=105, \sigma=0,3, \kappa=0,01, \theta=0,003$ y $\eta=0,1$, Intervalo de Confianza $=99 \%$

\begin{tabular}{|c|r|r|r|r|r|r|}
\hline \multirow{2}{*}{$\mathbf{N}$} & Iteraciones & $\begin{array}{c}\text { Error de } \\
\text { estimación }\end{array}$ & v Aproximado & $\begin{array}{c}\text { Tiempo } \\
\text { transcurrido } \\
\text { (segundos) }\end{array}$ & $\begin{array}{c}\text { Intervalo } \\
\text { superior }\end{array}$ & $\begin{array}{c}\text { Intervalo } \\
\text { inferior }\end{array}$ \\
\hline \multirow{2}{*}{1} & 1000 & 0.3727 & 7.1793 & 0.2357 & 7.9247 & 6.4339 \\
\cline { 2 - 8 } & 100000 & 0.0376 & 7.1719 & 22.7961 & 7.2471 & 7.0967 \\
\hline \multirow{2}{*}{4} & 1000 & 0.3976 & 7.4228 & 0.8234 & 8.218 & 6.6276 \\
\cline { 2 - 8 } & 100000 & 0.0406 & 7.2853 & 79.9360 & 7.3665 & 7.2041 \\
\hline \multirow{2}{*}{6} & 1000 & 0.3931 & 7.4876 & 1.2634 & 8.2738 & 6.7014 \\
\cline { 2 - 8 } & 100000 & 0.0411 & 7.2772 & 118.6203 & 7.3594 & 7.195 \\
\cline { 2 - 8 } & 1000 & 0.3956 & 7.0441 & 2.2839 & 7.8353 & 6.2529 \\
\hline \multirow{2}{*}{52} & 100000 & 0.0418 & 7.3730 & 230.2247 & 7.4566 & 7.2894 \\
\cline { 2 - 7 } & 100000 & 0.4297 & 7.2234 & 9.723 & 8.0828 & 6.364 \\
\hline
\end{tabular}

Gráfico 2. Vega aproximado con el Método de Trayectorias-Euler. Parámetros: $S 0=100, R_{f}=1 \%, T=1, m=\{1,4,6,12,52\}, K=105, \sigma=0,3, \kappa=0,01, \theta=0,003$ y $\eta=0,1$, Intervalo de Confianza $=99 \%$. Las estrellas representan la aproximación

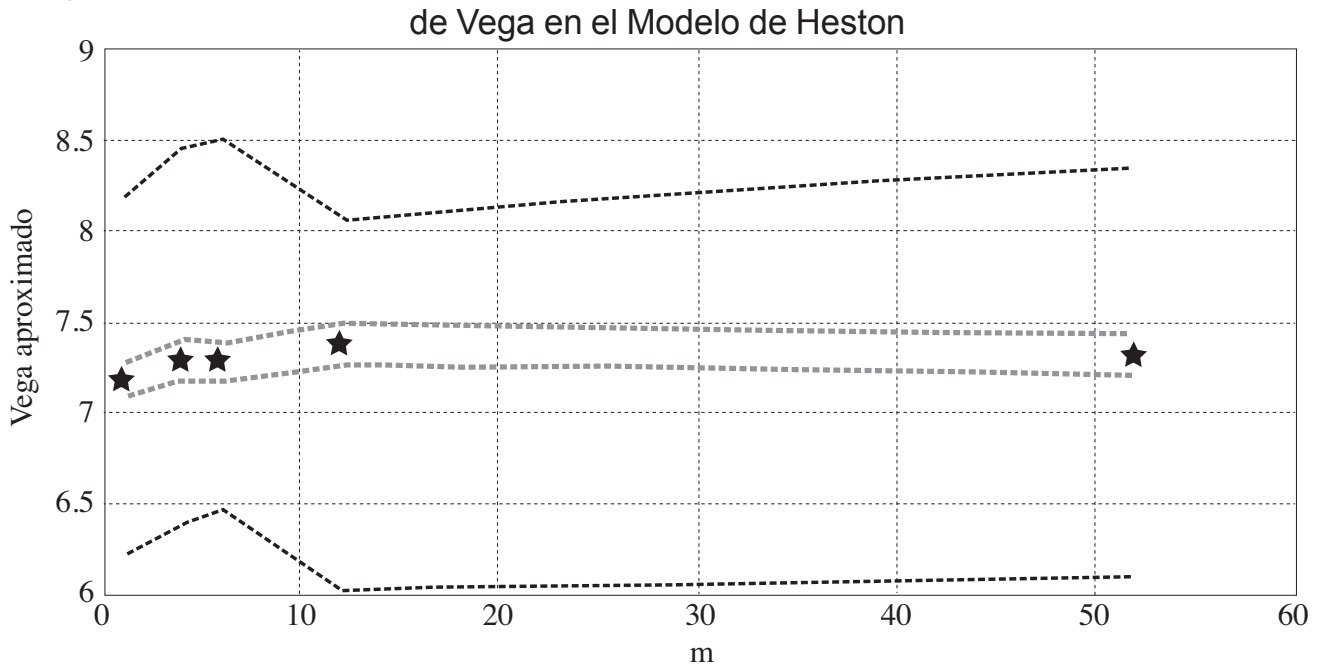


El gráfico 2 muestra que con 1.000 simulaciones para $m=12$, el Vega aproximado de Heston, construido con un intervalo de $99 \%$ de confianza, tiene un límite superior de 7,8353 y un límite inferior de 6,2529. Con 100.000 simulaciones y $m=12$, el Vega aproximado tiene un límite superior de 7,4566 y un límite inferior de 7,2894.

Para los otros casos que se establecen en la tabla 4 (casos 1,2, 3 y 4), en general se observa que con 100.000 simulaciones el Vega aproximado está en el intervalo construido con un $99 \%$ de confianza con límite superior promedio de 3,8642 y límite inferior promedio de 3,7038 para $m=12$ (sección 5.2).

Posteriormente, se realiza el experimento con 1.000 y 100.000 simulaciones con parámetros fijos: $S 0=100, R_{f}=1 \%, T=1, m=\{1,4,6,12,52\}, \sigma=0,3, K=$ 105 , y parámetros variables: $\kappa, \theta, \eta$, como se muestra en la tabla 6 .

El objetivo de este experimento es observar cómo se comporta el Vega aproximado por el Método de Trayectorias-Euler, ante cambios en $\kappa, \theta$ y $\eta$. Por ejemplo, ¿qué pasa cuando $\eta$ se incrementa?, ¿cómo influye este cambio en el precio del activo?

Tabla 6. Cambio en los parámetros $\mathrm{\kappa}, \theta$ y $\eta$, en el modelo de Heston

\begin{tabular}{|c|c|c|c|c|c|c|}
\hline Parámetro & Caso 1 & Caso 2 & Caso 3 & Caso 4 & Caso 5 & Caso 6 \\
\hline $\mathrm{K}$ & 105 & 105 & 105 & 105 & 105 & 105 \\
\hline$\sigma$ & 0.3 & 0.3 & 0.3 & 0.3 & 0.3 & 0.3 \\
\hline$\kappa$ & 0.03 & 0.003 & 0.01 & 0.01 & 0.01 & 0.01 \\
\hline$\eta$ & 0.003 & 0.003 & 0.005 & 0.001 & 0.003 & 0.003 \\
\hline$\eta$ & 0.1 & 0.1 & 0.1 & 0.1 & 0.2 & 0.05 \\
\hline $\begin{array}{l}\text { V Método Tra- } \\
\text { yectorias - Euler } \\
(100.000 \text { sim y } \\
\text { m=12) }\end{array}$ & 7.2915 & 7.4013 & 7.3731 & 7.3728 & $7.14 .6048-0.0006 \mathrm{i}$ & 3.6934 \\
\hline
\end{tabular}

$\mathrm{Al}$ realizar las simulaciones correspondientes se observan los siguientes cambios frente a variaciones ante cada uno de los parámetros móviles:

Variaciones en Kappa ( $\kappa)$. El caso 1 con parámetros fijos: $S 0=100, R_{f}=1 \%, T=$ 1 , con $m=\{1,4,6,12,52\}$, y parámetros variables: $K=105, \sigma=0,3, \kappa=0,03, \theta$ $=0,003$ y $\eta=0,1$, con un intervalo de confianza del $99 \%$, cuando se mueve hacia arriba pasando de 0,01 a 0,03 , con 1.000 simulaciones, el valor del Vega aproxi- 
mado por el Método de Trayectorias-Euler tiene un límite superior de 7,7461 y un límite inferior de 6,1793 para $m=12$. Con 100.000 simulaciones y $m=12$ el valor de Vega aproximado tiene un límite superior de 7,3743 e inferior de 7,2087, presentando una disminución frente al caso base de 1,1037\% en el límite superior y de $1.1071 \%$ en el límite inferior. Como se puede ver, el impacto en el valor del Vega aproximado ante un incremento en $\kappa$ es significativo para los casos evaluados.

Para el caso 2 con parámetros fijos: $S 0=100, R_{f}=1 \%, T=1$, con $m=\{1,4,6$, $12,52\}$, y parámetros variables: $K=105, \sigma=0,3, \kappa=0,003, \theta=0,003$ у $\eta=0,1$, con un intervalo de confianza del $99 \%$, al disminuir el valor de $\kappa$ (frente al caso base), con 1.000 simulaciones el límite superior del Vega aproximado es de 7,8613 y el límite inferior es de 6,2733 para $m=12$. Con 100.000 simulaciones y $m=12$ el límite superior del Vega aproximado aumenta en $0,3822 \%$, llegando a un valor de 7,4851, y el límite inferior se incrementa en $0,3855 \%$ con un valor de 7,3175 para $m=12$, luego las variaciones en este parámetro resultan ser significativas en el proceso de la aproximación del Vega de Heston para los casos evaluados.

Variaciones en Theta $(\theta)$. En el caso 3, con parámetros fijos: $S 0=100, R_{f}=1 \%$, $T=1$, con $m=\{1,4,6,12,52\}$, y parámetros variables: $K=105, \sigma=0,3, \kappa=0,01$, $\theta=0,005$ y $\eta=0,1$, con un intervalo de confianza del $99 \%$; cuando $\theta=0,005$ con 1.000 simulaciones los valores de los límites superior e inferior del Vega aproximado son de 7,8393 y 6,2489, y con 100.000 simulaciones los límites superior e inferior aumentan en 0,0013 y $0,0014 \%$ (respectivamente) frente al caso base para $m=12$.

Por otra parte, se observa que cuando el valor de $\theta$ disminuye (caso 4, parámetros fijos: $S 0=100, R_{f}=1 \%, T=1$, con $m=\{1,4,6,12,52\}$, y parámetros variables: $K=105, \sigma=0,3, \kappa=0,01, \theta=0,001$ у $\eta=0,1$, con un intervalo de confianza del 99\%), los límites superior e inferior del Vega aproximado con 100.000 simulaciones decrecen en $0,0027 \%$ respecto al caso base para $m=12$, por tanto, se concluye que este parámetro no es significativo para los casos evaluados.

Variaciones en la volatilidad de la volatilidad $(\eta)$. Al realizar el experimento en el caso 6, con parámetros fijos: $S 0=100, R_{f}=1 \%, T=1$, con $m=\{1,4,6,12$, $52\}$, y parámetros variables: $K=105, \sigma=0,3, \kappa=0,01, \theta=0,003$ y $\eta=0,05$, con un intervalo de confianza del $99 \%$, se encuentra que con 100.000 simulaciones cuando disminuye los límites superior e inferior del Vega aproximado decrecen sustancialmente en un 49,9128 y 49,8999\% respectivamente, es decir, el límite 
superior cae de 7,4566 a 3,7348, y el límite inferior cae de 7,2894 a 3,652 para $m=12$, luego este parámetro es el más significativo para los casos evaluados.

Posteriormente, al realizar el experimento en el caso 5 con parámetros fijos: $S 0$ $=100, R_{f}=1 \%, T=1$, con $m=\{1,4,6,12,52\}$, y parámetros variables: $K=105$, $\sigma=0,3, \kappa=0,01, \theta=0,003$ y $\eta=0,2$, con un intervalo de confianza del $99 \%$, se realiza la aproximación de Vega incrementando $\eta$ a 0,2 , en este caso específico se muestra que cuando $\eta$ aumenta, la varianza se acerca a cero y el proceso de discretización posiblemente la vuelve negativa (como se observa en el gráfico 3), lo que ocasiona que el método genere números imaginarios dada la $\sqrt{V_{t}}$ en la ecuación diferencial estocástica que representa como se puede ver en la ecuación 7.

Gráfico 3. Vega aproximado con el Método de Trayectorias-Euler. Parámetros: $S 0=100, R_{f}=0,01, T=1, m=\{1,4,6,12,52\}, K=105, \sigma=0,3, \kappa=0,01, \theta=0,003$ у $\eta=0,2$, Intervalo de Confianza $=99 \%$

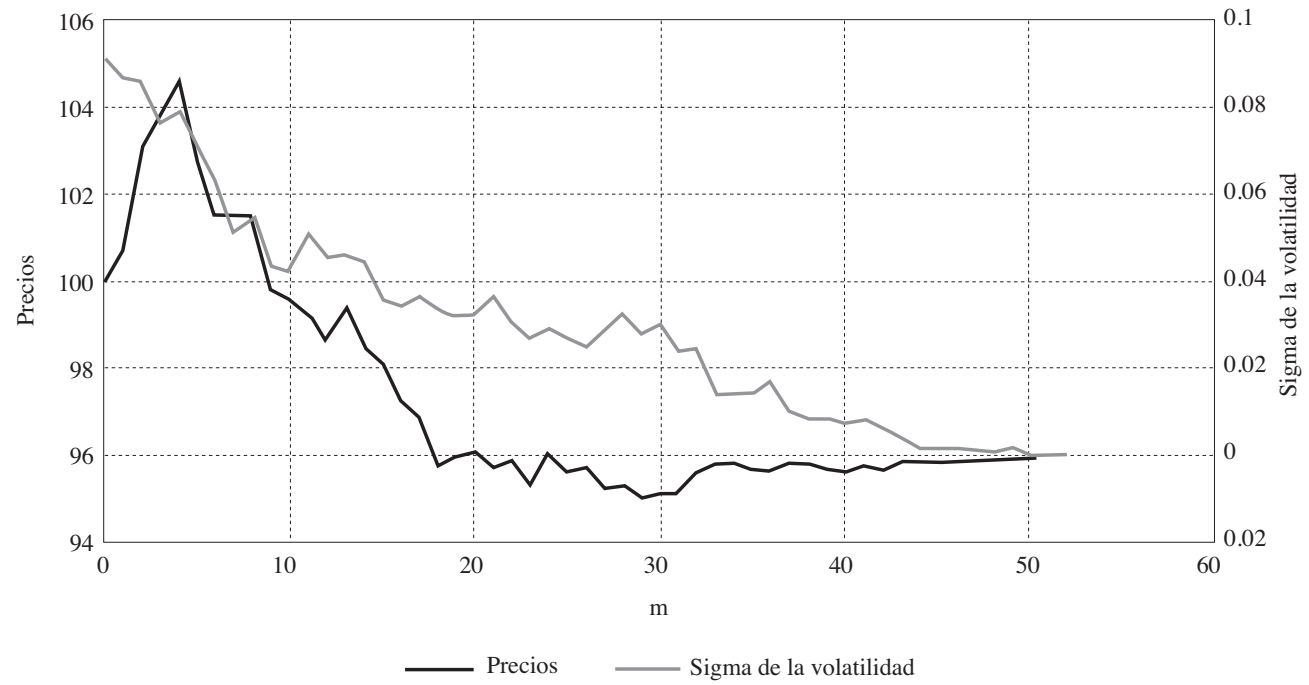

Según lo anterior, se concluye que el parámetro más significativo en la aproximación de Vega para el modelo de Heston es $\eta$.

\section{Conclusiones y extensiones}

En este trabajo se expuso el modelo de Black-Scholes, con énfasis especial en su supuesto de volatilidad constante; posteriormente, se mostró cómo se calcula Vega bajo este modelo. Ya que en el mercado real la volatilidad no es constante sino 
que fluctúa, se procedió a exponer diferentes modelos de volatilidad estocástica, entre ellos el modelo desarrollado por Heston (1993), que permitan hacer una aproximación de Vega.

Puesto que bajo el modelo de volatilidad estocástica de Heston la sensibilidad del valor de una opción ante cambios en $V_{0}$ no tiene solución exacta, se decidió implementar el Método de Trayectorias-Euler con diferencias finitas, para discretizar las ecuaciones diferenciales del modelo de Heston, simular los precios y realizar la aproximación de Vega.

Al implementar el Método de Trayectorias-Euler para hacer la aproximación de Vega en el Modelo de Black-Scholes, se observa que tiene una importancia alta puesto que en las primeras particiones se presenta sesgo en el Vega aproximado. Sin embargo, a medida que $m$ aumenta $(m \geq 12)$, el Vega de Black-Scholes cae perfectamente dentro del intervalo construido ya que hay un ajuste en el valor del Vega aproximado.

Respecto al Vega aproximado por el Método de Trayectorias-Euler en el Modelo de Heston, el cual se caracteriza por tener volatilidad estocástica, se observa que el parámetro más relevante en este proceso estocástico es $\eta$, ya que ante un incremento en este parámetro, la varianza se acerca a cero y el proceso de discretización posiblemente la vuelve negativa generando números imaginarios; por tanto, se plantea como posible extensión de este trabajo hacer una revisión sobre este caso específico y, de esta manera, hacer una mejora en el método cuando $\eta$ tiende a ser muy alta.

Se observa también que cuando $\eta$ disminuye, decrece sustancialmente el valor del Vega aproximado, en donde los límites superior e inferior del mismo caen en 49,9128 y 49,8999\% respectivamente (frente al caso base), confirmando que $\eta$ es el parámetro más relevante en la aproximación de Vega por el Método de Trayectorias-Euler.

Finalmente, en vista de que este trabajo se desarrolló utilizando correlación cero, también se plantea como posible tema de investigación averiguar cómo se ve afectado el valor de Vega ante cambios en el parámetro de correlación. 


\section{Referencias}

Chen, H.-Y., Lee, C.-F. y Shih, W. (n.d.). Derivation and Application of greek letters. New Jersey.

Glasserman, P. (2003). Monte Carlo Methods in Financial Engineering. New York.

González, A. (2008). Análisis empírico de la volatilidad estocástica y saltos para modelos en tiempo continuo de índices bursátiles utilizando el EMM. Universidad del País Vasco.

Hull, J. C. (2012). Options, futures and other derivatives (8th ed.). New Jersey: Pearson. León, C. (2009). Borradores de Economía, 570. Bogotá: Banco de La República.

Medina, R. T. (2010). Una revisión de los modelos de volatilidad estocástica. Comunicaciones en Estadística, 3 (1), 20.

Moodley, N. (2005). The Heston Model : A Practical Approach with Matlab Code. Johannesburg.

Moreno, J. F. (2012). Valoración de Activos contingentes y medidas martingala equivalentes. Cuadernos del CIPE, 13.

Quintero Sánchez, J. E. (2011). La volatilidad en los mercados financieros, una muetra de incertidmbre y especulación. Bogotá.

Ramírez, A. O., Martínez, F. V. y Herrera, F. L. (n.d.). Una nota sobre la sensibilidad de los parámetros del modelo de volatilidad estocástica de Heston. Quantitativa Revista de Economía, 1 (1). 


\section{Anexos}

\subsection{Modelo Black - Scholes}

Anexo 1. Vega aproximado con el Método de Trayectorias-Euler. Parámetros: SO $=100, R_{f}=1 \%, T=1, m=\{1,4,6,12,52\}, K=105, \sigma=0,1$, Intervalo de Confianza $=99 \%$

\begin{tabular}{|c|r|r|r|r|r|r|r|}
\hline $\mathbf{N}$ & Iteraciones & $\mathbf{v}$ Analítico & $\begin{array}{c}\text { Error de } \\
\text { estimación }\end{array}$ & $\mathbf{v}$ Aproximado & $\begin{array}{c}\text { Tiempo } \\
\text { transcurrido } \\
\text { (segundos) }\end{array}$ & $\begin{array}{c}\text { Intervalo } \\
\text { superior }\end{array}$ & $\begin{array}{c}\text { Intervalo } \\
\text { inferior }\end{array}$ \\
\hline \multirow{2}{*}{1} & 1000 & 37.6805 & 1.9073 & 37.6141 & 1.0881 & 42.57308 & 32.65512 \\
\cline { 2 - 8 } & 100000 & 37.6805 & 0.1938 & 37.6991 & 12.2309 & 38.20298 & 37.19522 \\
\hline \multirow{2}{*}{4} & 1000 & 37.6805 & 2.0176 & 35.7056 & 0.4403 & 40.95136 & 30.45984 \\
\cline { 2 - 9 } & 100000 & 37.6805 & 0.2113 & 38.7403 & 41.7536 & 39.28968 & 38.19092 \\
\hline \multirow{2}{*}{6} & 1000 & 37.6805 & 2.0093 & 35.7252 & 0.6155 & 40.94938 & 30.50102 \\
\cline { 2 - 9 } & 100000 & 37.6805 & 0.2127 & 38.7625 & 61.7156 & 39.31552 & 38.20948 \\
\hline \multirow{2}{*}{12} & 1000 & 37.6805 & 2.058 & 37.3426 & 1.2093 & 42.6934 & 31.9918 \\
\cline { 2 - 8 } & 100000 & 37.6805 & 0.215 & 38.8312 & 120.7606 & 39.3902 & 38.2722 \\
\hline \multirow{2}{*}{52} & 1000 & 37.6805 & 2.1949 & 37.5021 & 5.2751 & 43.20884 & 31.79536 \\
\cline { 2 - 8 } & 100000 & 37.6805 & 0.2170 & 39.0492 & 532.7949 & 39.6134 & 38.485 \\
\hline
\end{tabular}

Anexo 2. Gráfico Vega aproximado con el Método de Trayectorias-Euler.

Parámetros: $S 0=100, R_{f}=1 \%, T=1, m=\{1,4,6,12,52\}, K=105, \sigma=0,1$, Intervalo de Confianza $=99 \%$. Las estrellas representan el valor analítico de Vega.

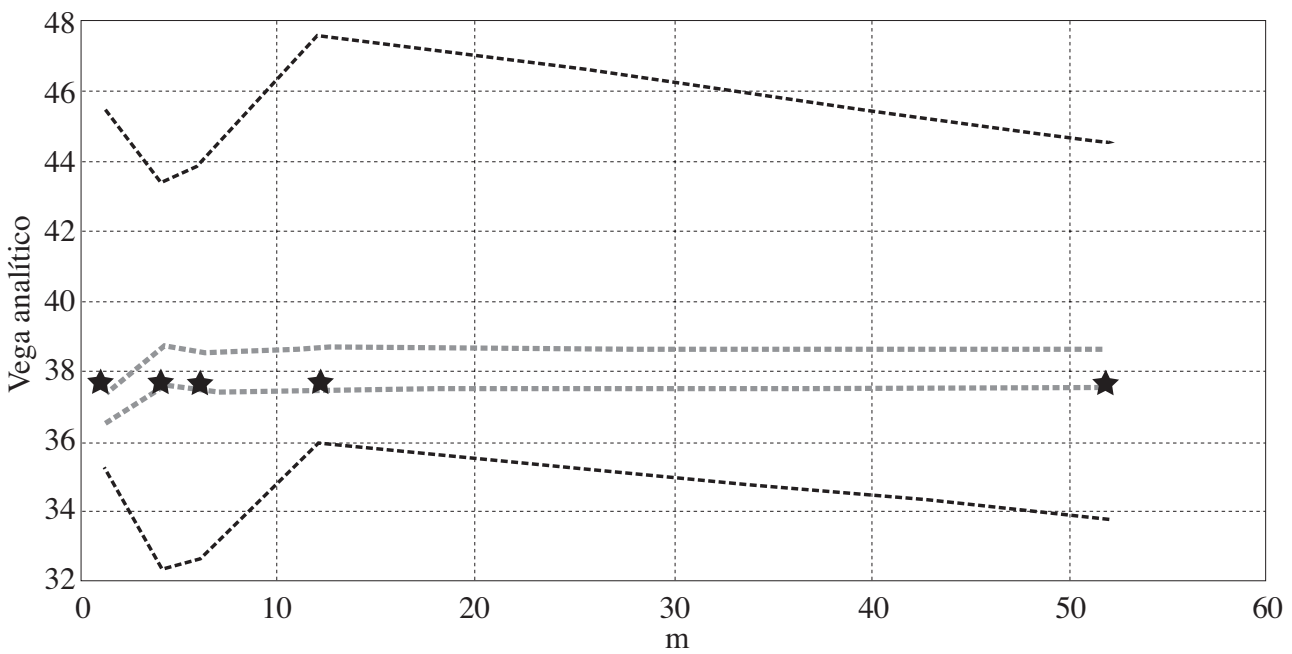


Anexo 3. Vega aproximado con el Método de Trayectorias-Euler.

Parámetros: $S 0=100, R_{f}=1 \%, T=1, m=\{1,4,6,12,52\}, K=105, \sigma=0,5$, Intervalo de Confianza $=99 \%$

\begin{tabular}{|c|r|r|r|r|r|r|r|}
\hline $\mathbf{N}$ & Iteraciones & $\mathbf{v}$ Analítico & $\begin{array}{c}\text { Error de } \\
\text { estimación }\end{array}$ & $\mathbf{v}$ Aproximado & $\begin{array}{c}\text { Tiempo } \\
\text { transcurrido } \\
\text { (segundos) }\end{array}$ & $\begin{array}{c}\text { Intervalo } \\
\text { superior }\end{array}$ & $\begin{array}{c}\text { Intervalo } \\
\text { inferior }\end{array}$ \\
\hline \multirow{2}{*}{1} & 1000 & 39.3056 & 1.7996 & 39.5491 & 0.1268 & 44.22806 & 34.87014 \\
\cline { 2 - 9 } & 100000 & 39.3056 & 0.1833 & 39.3572 & 12.2881 & 39.83378 & 38.88062 \\
\hline \multirow{2}{*}{4} & 1000 & 39.3056 & 2.8207 & 37.4048 & 0.4234 & 44.73862 & 30.07098 \\
\cline { 2 - 9 } & 100000 & 39.3056 & 0.2947 & 42.0300 & 42.0452 & 42.79622 & 41.26378 \\
\hline \multirow{2}{*}{6} & 1000 & 39.3056 & 2.7746 & 36.2497 & 0.6186 & 43.46366 & 29.03574 \\
\cline { 2 - 9 } & 100000 & 39.3056 & 0.3084 & 41.1979 & 61.4117 & 41.99974 & 40.39606 \\
\hline \multirow{2}{*}{12} & 1000 & 39.3056 & 2.9624 & 37.2871 & 1.2006 & 44.98934 & 29.58486 \\
\cline { 2 - 9 } & 100000 & 39.3056 & 0.3282 & 40.3717 & 119.5526 & 41.22502 & 39.51838 \\
\hline \multirow{2}{*}{52} & 1000 & 39.3056 & 3.5137 & 39.7773 & 5.692 & 48.91292 & 30.64168 \\
\cline { 2 - 8 } & 100000 & 39.3056 & 0.3403 & 39.6866 & 609.9478 & 40.57138 & 38.80182 \\
\hline
\end{tabular}

Anexo 4. Gráfico Vega aproximado con el Método de Trayectorias-Euler.

Parámetros: $S 0=100, R_{f}=1 \%, T=1, m=\{1,4,6,12,52\}, K=105, \sigma=0,5$, Intervalo de Confianza $=99 \%$. Las estrellas representan el valor analítico de Vega

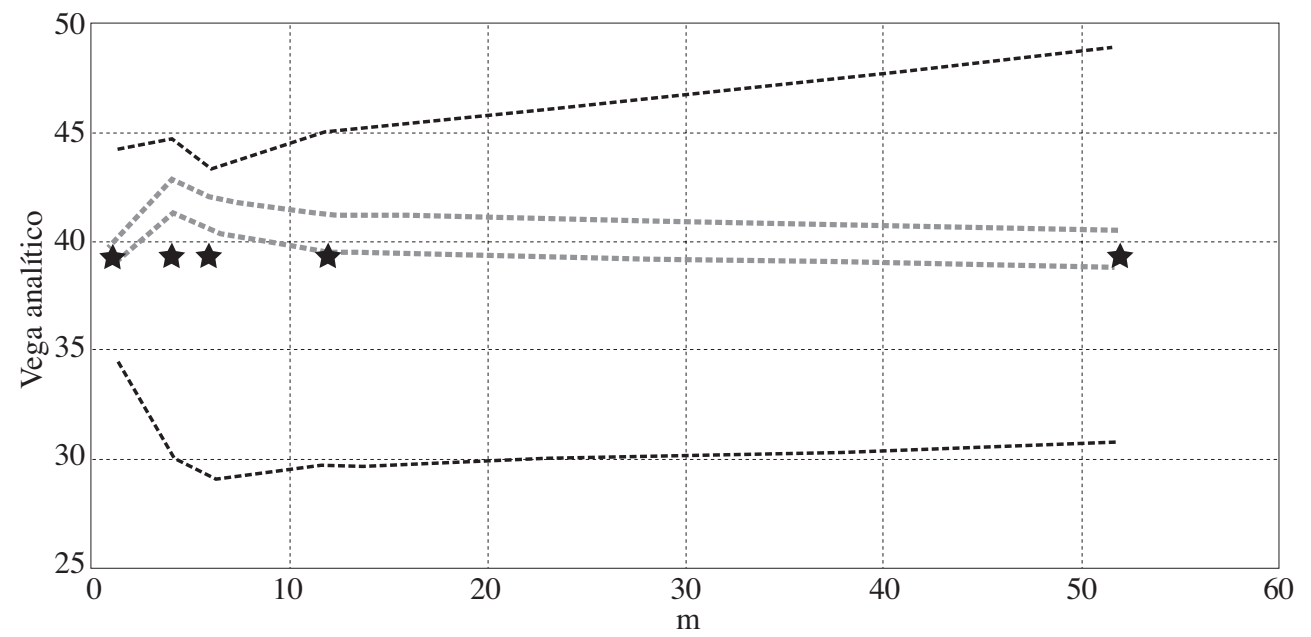


Anexo 5. Vega aproximado con el Método de Trayectorias-Euler.

Parámetros: $S 0=100, R_{f}=1 \%, T=1, m=\{1,4,6,12,52\}, K=105, \sigma=0,3$, Intervalo de Confianza $=99 \%$

\begin{tabular}{|c|r|r|r|r|r|r|r|}
\hline $\mathbf{N}$ & Iteraciones & $\mathbf{v}$ Analítico & $\begin{array}{c}\text { Error de } \\
\text { estimación }\end{array}$ & $\mathbf{v}$ Aproximado & $\begin{array}{c}\text { Tiempo } \\
\text { transcurrido } \\
\text { (segundos) }\end{array}$ & $\begin{array}{c}\text { Intervalo } \\
\text { superior }\end{array}$ & $\begin{array}{c}\text { Intervalo } \\
\text { inferior }\end{array}$ \\
\hline \multirow{2}{*}{1} & 1000 & 34.5832 & 1.8665 & 37.1945 & 0.1265 & 42.0474 & 32.3416 \\
\cline { 2 - 9 } & 100000 & 34.5832 & 0.1901 & 36.9095 & 11.9472 & 37.40376 & 36.41524 \\
\hline \multirow{2}{*}{4} & 1000 & 34.5832 & 2.4084 & 32.821 & 0.4261 & 39.08284 & 26.55916 \\
\cline { 2 - 9 } & 100000 & 34.5832 & 0.2535 & 36.0781 & 42.0221 & 36.7372 & 35.419 \\
\hline \multirow{2}{*}{6} & 1000 & 34.5832 & 2.4216 & 30.8877 & 0.6212 & 37.18386 & 24.59154 \\
\cline { 2 - 9 } & 100000 & 34.5832 & 0.2597 & 35.5308 & 61.9683 & 36.20602 & 34.85558 \\
\hline \multirow{2}{*}{12} & 1000 & 34.5832 & 2.5073 & 32.8986 & 1.3042 & 39.41758 & 26.37962 \\
\cline { 2 - 9 } & 100000 & 34.5832 & 0.2684 & 35.0549 & 121.459 & 35.75274 & 34.35706 \\
\hline \multirow{2}{*}{52} & 1000 & 34.5832 & 2.8115 & 33.5144 & 5.5625 & 40.8243 & 26.2045 \\
\cline { 2 - 8 } & 100000 & 34.5832 & 0.2745 & 34.8244 & 581.3868 & 35.5381 & 34.1107 \\
\hline
\end{tabular}

Anexo 6. Gráfico Vega aproximado con el Método de Trayectorias-Euler.

Parámetros: $S 0=100, R_{f}=1 \%, T=1, m=\{1,4,6,12,52\}, K=105, \sigma=0,3$, Intervalo de Confianza $=99 \%$. Las estrellas representan el valor analítico de Vega

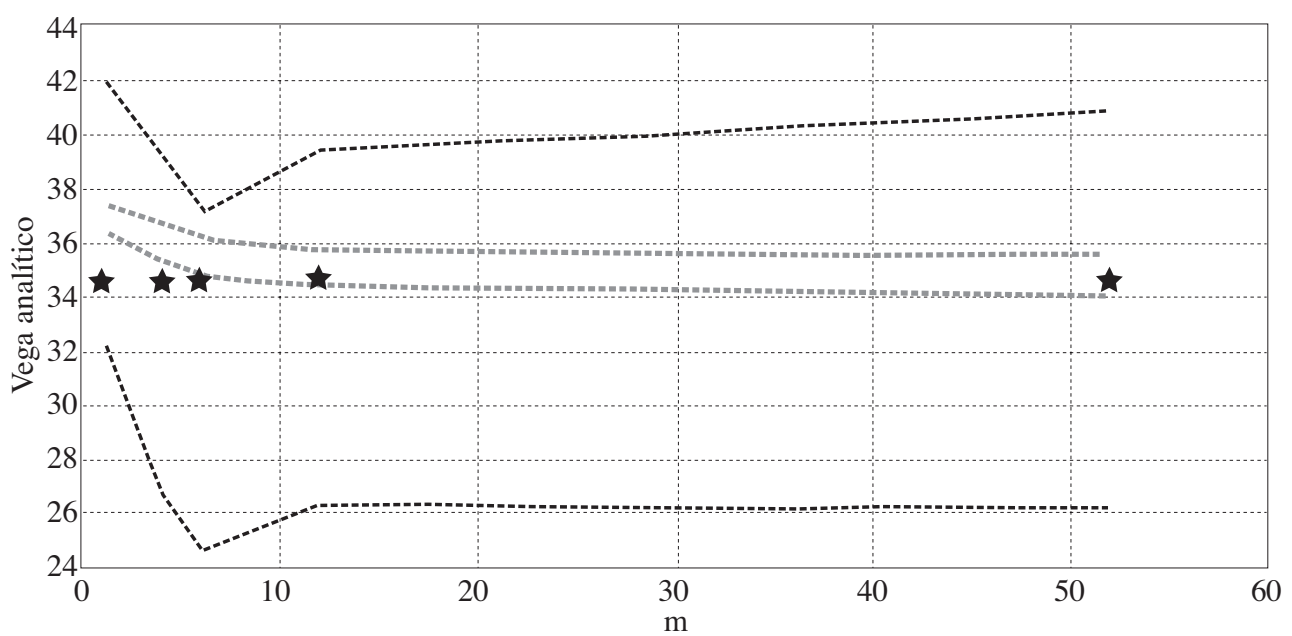


Anexo 7. Vega aproximado con el Método de Trayectorias-Euler. Parámetros:

$S 0=100, R_{f}=1 \%, T=1, m=\{1,4,6,12,52\}, K=120, \sigma=0,3$, Intervalo de Confianza $=99 \%$

\begin{tabular}{|c|r|r|r|r|r|r|r|}
\hline $\mathbf{N}$ & Iteraciones & $\mathbf{v}$ Analítico & $\begin{array}{c}\text { Error de } \\
\text { estimación }\end{array}$ & $\mathbf{v}$ Aproximado & $\begin{array}{c}\text { Tiempo } \\
\text { transcurrido } \\
\text { (segundos) }\end{array}$ & $\begin{array}{c}\text { Intervalo } \\
\text { superior }\end{array}$ & $\begin{array}{c}\text { Intervalo } \\
\text { inferior }\end{array}$ \\
\hline \multirow{2}{*}{1} & 1000 & 36.4584 & 1.8628 & 32.0452 & 0.126 & 36.88848 & 27.20192 \\
\cline { 2 - 9 } & 100000 & 36.4584 & 0.1891 & 32.3010 & 11.9601 & 32.79266 & 31.80934 \\
\hline \multirow{2}{*}{4} & 1000 & 36.4584 & 2.349 & 31.8828 & 0.4237 & 37.9902 & 25.7754 \\
\cline { 2 - 9 } & 100000 & 36.4584 & 0.2465 & 36.2954 & 41.8303 & 36.9363 & 35.6545 \\
\hline \multirow{2}{*}{6} & 1000 & 36.4584 & 2.3299 & 32.3974 & 0.6177 & 38.45514 & 26.33966 \\
\cline { 2 - 9 } & 100000 & 36.4584 & 0.2523 & 36.3555 & 61.2696 & 37.01148 & 35.69952 \\
\hline \multirow{2}{*}{12} & 1000 & 36.4584 & 2.4253 & 34.1419 & 1.2027 & 40.44768 & 27.83612 \\
\cline { 2 - 9 } & 100000 & 36.4584 & 0.2608 & 36.4539 & 122.392 & 37.13198 & 35.77582 \\
\hline \multirow{2}{*}{52} & 1000 & 36.4584 & 2.7245 & 36.1666 & 5.8762 & 43.2503 & 29.0829 \\
\cline { 2 - 9 } & 100000 & 36.4584 & 0.2669 & 36.5264 & 629.9685 & 37.22034 & 35.83246 \\
\hline
\end{tabular}

Anexo 8. Gráfico Vega aproximado con el Método de Trayectorias-Euler.

Parámetros: $S 0=100, R_{f}=1 \%, T=1, m=\{1,4,6,12,52\}, K=120, \sigma=0,3$, Intervalo de Confianza $=99 \%$. Las estrellas representan el valor analítico de Vega

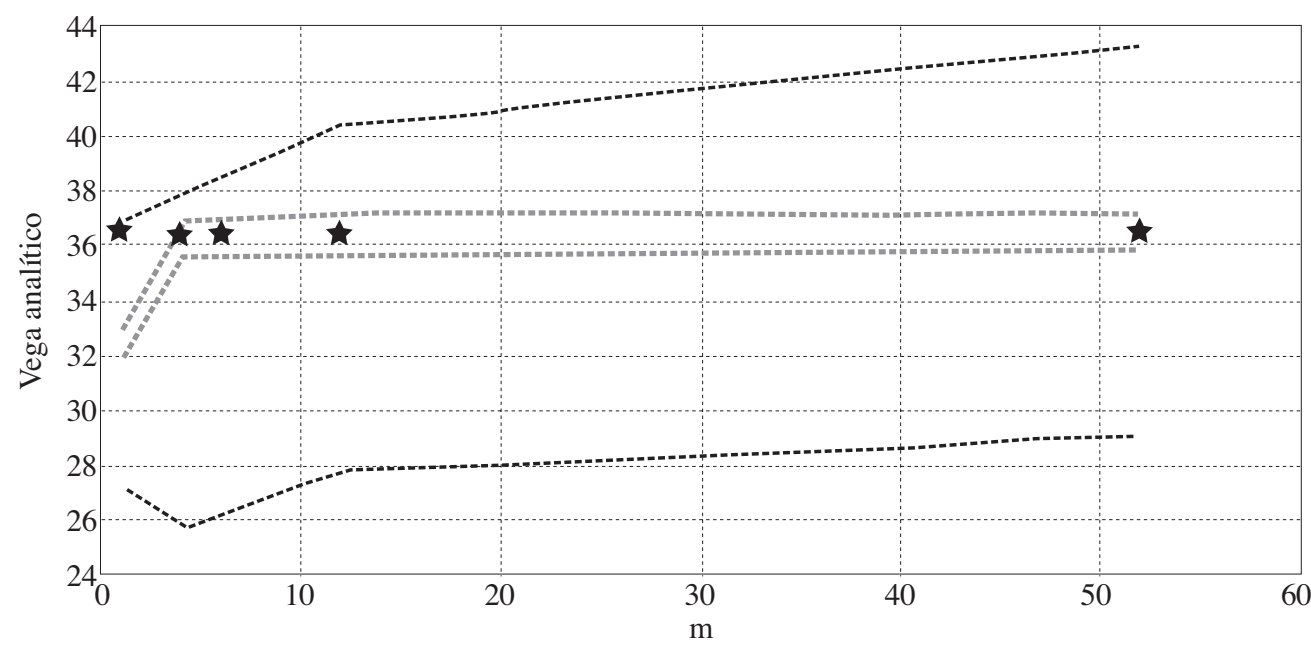




\subsubsection{Cuadro resumen de resultados del Vega aproximado utilizando el método de Trayectorias-Euler en el modelo de Black-Scholes}

Anexo 9. Cuadro resumen de resultados utilizando los parámetros establecidos en la tabla 2. Comparación del Vega analítico y el Vega generado por simulación con el Método de Trayectorias-Euler para $m=12$

\begin{tabular}{|c|c|c|c|c|c|}
\hline & \multicolumn{2}{|c|}{ Parámetros } & \multirow{2}{*}{$\begin{array}{l}\text { Número de } \\
\text { simulaciones }\end{array}$} & \multirow{2}{*}{ Vega analítico } & \multirow{2}{*}{$\begin{array}{l}\text { Vega Método Tra- } \\
\text { yectorias - Euler }\end{array}$} \\
\hline $\mathbf{N}$ & $\kappa$ & $\square$ & & & \\
\hline \multirow{2}{*}{1} & \multirow{2}{*}{90} & \multirow{2}{*}{0.3} & 1.000 & 34.5832 & 32.8986 \\
\hline & & & 100.000 & 34.5832 & 35.0549 \\
\hline \multirow{2}{*}{2} & \multirow{2}{*}{105} & \multirow{2}{*}{0.3} & 1.000 & 39.8857 & 38.1410 \\
\hline & & & 100.000 & 39.8857 & 40.1607 \\
\hline \multirow{2}{*}{3} & \multirow{2}{*}{105} & \multirow{2}{*}{0.1} & 1.000 & 37.6805 & 37.3426 \\
\hline & & & 100.000 & 37.6805 & 38.8312 \\
\hline \multirow{2}{*}{4} & \multirow{2}{*}{105} & \multirow{2}{*}{0.5} & 1.000 & 39.3056 & 37.2871 \\
\hline & & & 100.000 & 39.3056 & 40.3717 \\
\hline \multirow{2}{*}{5} & \multirow{2}{*}{120} & \multirow{2}{*}{0.3} & 1.000 & 36.4584 & 34.1419 \\
\hline & & & 100.000 & 36.4584 & 36.4539 \\
\hline
\end{tabular}

\subsection{Modelo Heston}

Anexo 10. Vega aproximado con el Método de Trayectorias-Euler. Caso base 3. Parámetros: $S 0=100, R_{f}=1 \%, T=1, m=\{1,4,6,12,52\}, K=90, \sigma=0,3, \kappa=0,01$, $\theta=0,003$ y $\eta=0,1$, Intervalo de Confianza $=99 \%$

\begin{tabular}{|c|r|r|r|r|r|r|}
\hline \multirow{2}{*}{$\mathbf{N}$} & Iteraciones & $\begin{array}{c}\text { Error de } \\
\text { estimación }\end{array}$ & v Aproximado & $\begin{array}{c}\text { Tiempo } \\
\text { transcurrido } \\
\text { (segundos) }\end{array}$ & $\begin{array}{c}\text { Intervalo } \\
\text { superior }\end{array}$ & $\begin{array}{c}\text { Intervalo } \\
\text { inferior }\end{array}$ \\
\hline \multirow{2}{*}{1} & 1000 & 0.4948 & 3.8089 & 0.2309 & 4.7985 & 2.8193 \\
\cline { 2 - 7 } & 100000 & 0.0495 & 3.7545 & 22.6980 & 3.8535 & 3.6555 \\
\hline \multirow{2}{*}{4} & 1000 & 0.5076 & 4.1593 & 0.803 & 5.1745 & 3.1441 \\
\cline { 2 - 7 } & 100000 & 0.0528 & 3.3910 & 80.3264 & 3.4966 & 3.2854 \\
\hline \multirow{2}{*}{6} & 1000 & 0.5226 & 3.5442 & 1.4981 & 4.5894 & 2.499 \\
\hline \multirow{2}{*}{12} & 100000 & 0.0532 & 3.3449 & 117.5652 & 3.4513 & 3.2385 \\
\cline { 2 - 7 } & 100000 & 0.0538 & 3.4250 & 229.8570 & 3.5326 & 3.3174 \\
\hline \multirow{2}{*}{52} & 1000 & 0.546 & 3.2967 & 10.0595 & 4.3887 & 2.2047 \\
\cline { 2 - 7 } & 100000 & 0.0541 & 3.3199 & 1032.0000 & 3.4281 & 3.2117 \\
\hline
\end{tabular}


Anexo 11. Gráfico Vega aproximado con el Método de Trayectorias-Euler. Caso base 3.

Parámetros: $S 0=100, R_{f}=1 \%, T=1, m=\{1,4,6,12,52\}, K=90, \sigma=0,3, \kappa=0,01$,

$\theta=0,003$ y $\eta=0,1$, Intervalo de Confianza $=99 \%$. Las estrellas representan la aproximación de Vega en el modelo de Heston

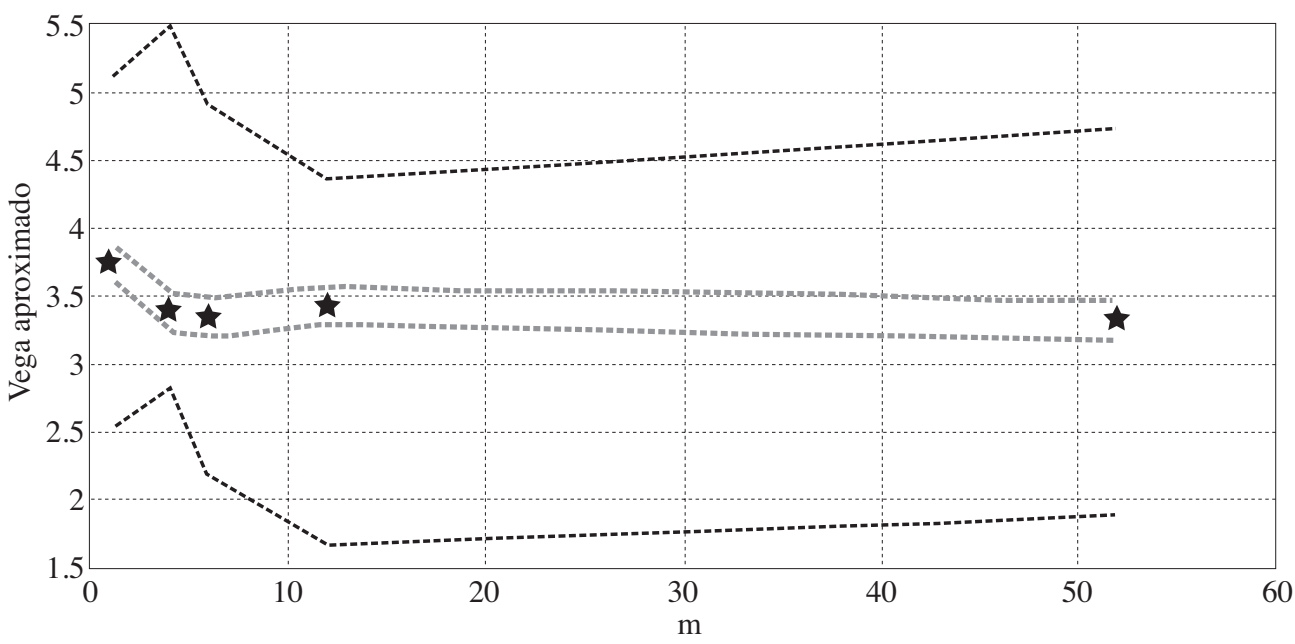

Anexo 12. Vega aproximado con el Método de Trayectorias-Euler. Caso base 4, Parámetros: $S 0=100, R_{f}=1 \%, T=1, m=\{1,4,6,12,52\}, K=120, \sigma=0,3, \kappa=0,01$, $\theta=0,003$ y $\eta=0,1$, Intervalo de Confianza $=99 \%$

\begin{tabular}{|c|r|r|r|r|r|r|}
\hline \multirow{2}{*}{$\mathbf{N}$} & Iteraciones & $\begin{array}{c}\text { Error de } \\
\text { estimación }\end{array}$ & v Aproximado & $\begin{array}{c}\text { Tiempo } \\
\text { transcurrido } \\
\text { (segundos) }\end{array}$ & $\begin{array}{c}\text { Intervalo } \\
\text { superior }\end{array}$ & $\begin{array}{c}\text { Intervalo } \\
\text { inferior }\end{array}$ \\
\hline \multirow{2}{*}{1} & 1000 & 0.1911 & 0.7108 & 0.2543 & 1.093 & 0.3286 \\
\cline { 2 - 7 } & 100000 & 0.0204 & 0.8461 & 22.5498 & 0.8869 & 0.8053 \\
\hline \multirow{2}{*}{4} & 1000 & 0.2152 & 0.9093 & 0.8317 & 1.3397 & 0.4789 \\
\cline { 2 - 8 } & 100000 & 0.0265 & 1.3204 & 79.6495 & 1.3734 & 1.2674 \\
\hline \multirow{2}{*}{6} & 1000 & 0.2404 & 1.0643 & 1.1535 & 1.5451 & 0.5835 \\
\cline { 2 - 7 } & 100000 & 0.0275 & 1.4007 & 118.4848 & 1.4557 & 1.3457 \\
\hline \multirow{2}{*}{12} & 1000 & 0.2491 & 1.1549 & 2.3164 & 1.6531 & 0.6567 \\
\hline \multirow{2}{*}{52} & 100000 & 0.0286 & 1.4722 & 230.7367 & 1.5294 & 1.415 \\
\cline { 2 - 7 } & 1000 & 0.3081 & 1.5628 & 10.8055 & 2.179 & 0.9466 \\
\hline \multirow{2}{*}{5} & 10000 & 0.0294 & 1.5405 & 1101.5 & 1.5993 & 1.4817 \\
\hline
\end{tabular}


Anexo 13. Gráfico Vega aproximado con el Método de Trayectorias-Euler. Caso base

4. Parámetros: $S 0=100, R_{f}=1 \%, T=1, m=\{1,4,6,12,52\}, K=120, \sigma=0,3$, $\kappa=0,01, \theta=0,003$ у $\eta=0,1$, Intervalo de Confianza $=99 \%$. Las estrellas representan la aproximación de Vega en el modelo de Heston

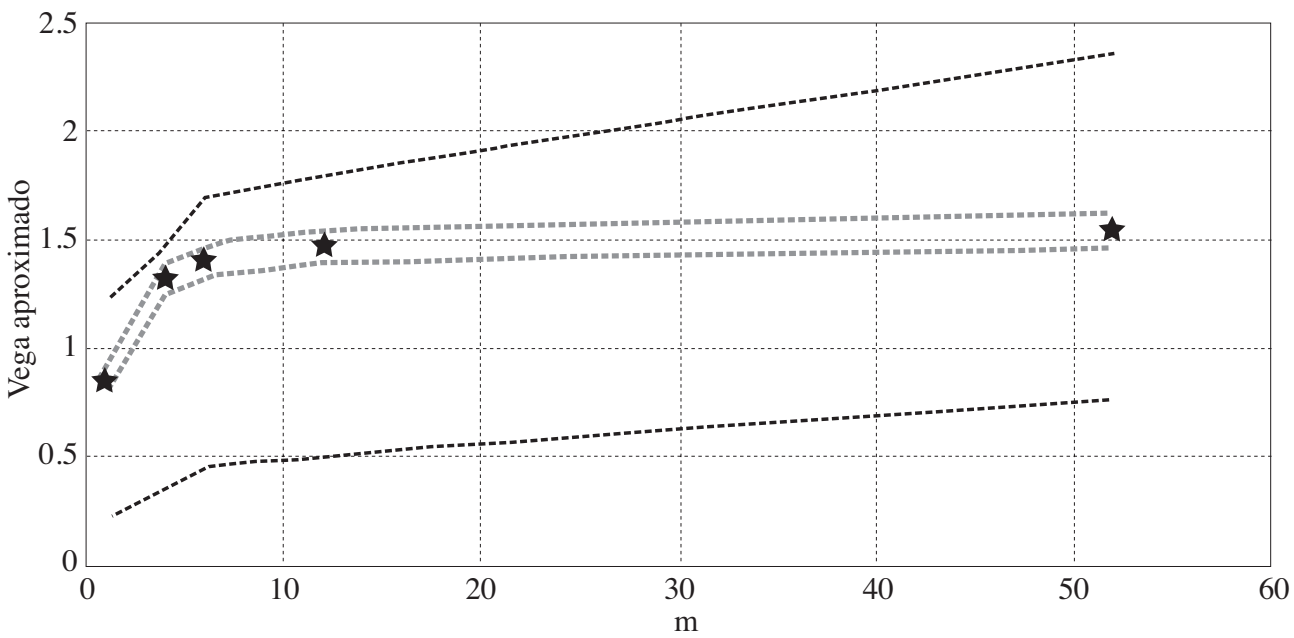

Anexo 14. Vega aproximado con el Método de Trayectorias-Euler. Caso 6 (cambios). Parámetros: $S 0=100, R_{f}=1 \%, T=1, m=\{1,4,6,12,52\}, K=105, \sigma=0,3, \kappa=0,01$, $\theta=0,003$ y $\eta=0,05$, Intervalo de Confianza $=99 \%$

\begin{tabular}{|c|r|r|r|r|r|r|}
\hline \multirow{2}{*}{$\mathbf{N}$} & Iteraciones & $\begin{array}{c}\text { Error de } \\
\text { estimación }\end{array}$ & v Aproximado & $\begin{array}{c}\text { Tiempo } \\
\text { transcurrido } \\
\text { (segundos) }\end{array}$ & $\begin{array}{c}\text { Intervalo } \\
\text { superior }\end{array}$ & $\begin{array}{c}\text { Intervalo } \\
\text { inferior }\end{array}$ \\
\hline \multirow{2}{*}{1} & 1000 & 0.1864 & 3.5896 & 0.3106 & 3.9624 & 3.2168 \\
\cline { 2 - 7 } & 100000 & 0.0188 & 3.5859 & 30.0678 & 3.6235 & 3.5483 \\
\hline \multirow{2}{*}{4} & 1000 & 0.2003 & 3.7678 & 1.0822 & 4.1684 & 3.3672 \\
\cline { 2 - 8 } & 100000 & 0.0203 & 3.6500 & 107.3873 & 3.6904 & 3.6096 \\
\hline \multirow{2}{*}{6} & 1000 & 0.1958 & 3.7596 & 1.5847 & 4.1512 & 3.368 \\
\cline { 2 - 8 } & 100000 & 0.0204 & 3.6470 & 157.7334 & 3.6878 & 3.6062 \\
\hline \multirow{2}{*}{12} & 1000 & 0.1953 & 3.5108 & 3.1398 & 3.9014 & 3.1202 \\
\hline \multirow{2}{*}{52} & 100000 & 0.0207 & 3.6934 & 309.6048 & 3.7348 & 3.652 \\
\cline { 2 - 7 } & 1000 & 0.2099 & 3.5621 & 9.7319 & 3.9819 & 3.1423 \\
\hline \multirow{2}{*}{5} & 100000 & 0.0209 & 3.6734 & 981.0148 & 3.7152 & 3.6316 \\
\hline
\end{tabular}


Anexo 15. Gráfico Vega aproximado con el Método de Trayectorias-Euler. Caso 6 (cambios). Parámetros: $S 0=100, R_{f}=1 \%, T=1, m=\{1,4,6,12,52\}, K=105, \sigma=0,3$, $\kappa=0,01, \theta=0,003$ y $\eta=0,05$, Intervalo de Confianza $=99 \%$. Las estrellas representan la aproximación de Vega en el modelo de Heston

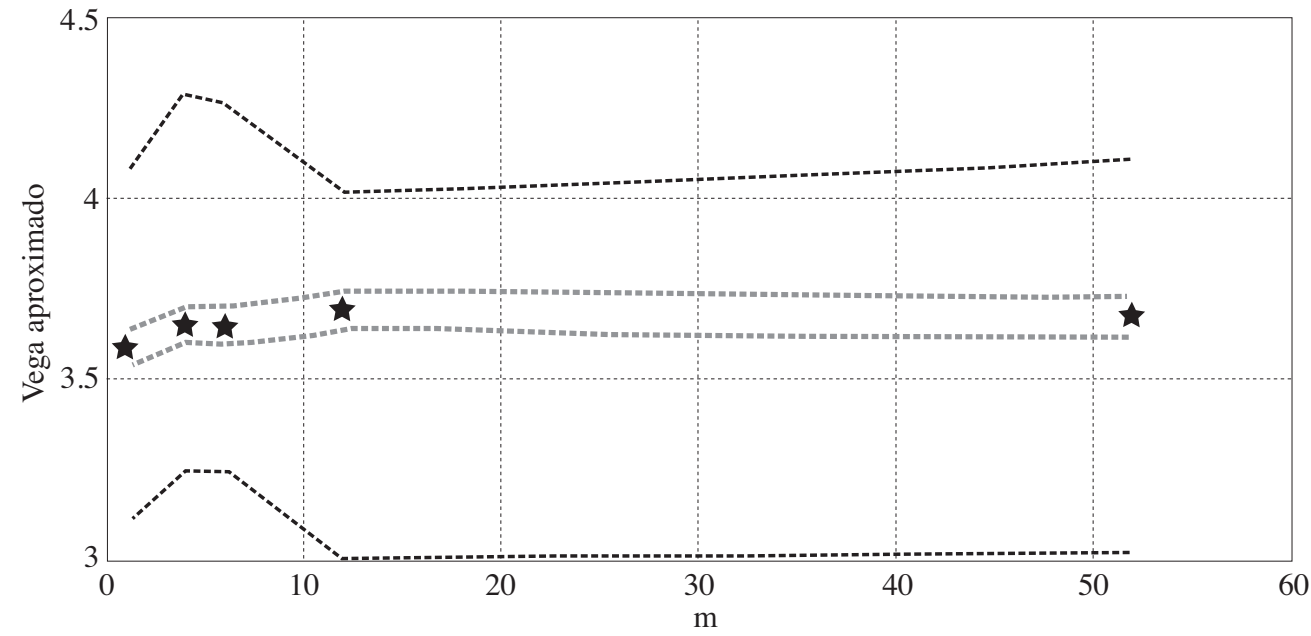

5.2.1. Cuadro resumen de resultados del Vega aproximado utilizando el Método de Trayectorias-Euler en el modelo de Heston

Anexo 16. Cuadro resumen de resultados del Vega generado por simulación con el Método de Trayectorias-Euler para $=12$

\begin{tabular}{|c|c|c|c|c|c|c|c|}
\hline \multirow{2}{*}{ N..} & \multicolumn{5}{|c|}{ Parámetros } & \multirow{2}{*}{$\begin{array}{l}\text { Número } \\
\text { simulaciones }\end{array}$} & \multirow{2}{*}{$\begin{array}{l}\text { Vega generado } \\
\text { por el Método de } \\
\text { Trayectoria - Euler }\end{array}$} \\
\hline & $\mathbf{K}$ & $\square$ & $\kappa$ & $\theta$ & $\eta$ & & \\
\hline \multirow{2}{*}{1} & \multirow{2}{*}{105} & \multirow{2}{*}{0.3} & \multirow{2}{*}{0.1} & \multirow{2}{*}{0.003} & \multirow{2}{*}{0.1} & 1.000 & 7.0441 \\
\hline & & & & & & 100.000 & 7.3730 \\
\hline \multirow{2}{*}{2} & \multirow{2}{*}{105} & \multirow{2}{*}{0.15} & \multirow{2}{*}{0.01} & \multirow{2}{*}{0.003} & \multirow{2}{*}{0.1} & 1.000 & 2.1678 \\
\hline & & & & & & 100.000 & 2.2657 \\
\hline \multirow{2}{*}{3} & \multirow{2}{*}{105} & \multirow{2}{*}{0.5} & \multirow{2}{*}{0.01} & \multirow{2}{*}{0.003} & \multirow{2}{*}{0.1} & 1.000 & 7.4963 \\
\hline & & & & & & 100.000 & 8.0264 \\
\hline \multirow{2}{*}{4} & \multirow{2}{*}{90} & \multirow{2}{*}{0.3} & \multirow{2}{*}{0.1} & \multirow{2}{*}{0.003} & \multirow{2}{*}{0.1} & 1.000 & 3.0226 \\
\hline & & & & & & 100.000 & 3.4250 \\
\hline \multirow{2}{*}{5} & \multirow{2}{*}{120} & \multirow{2}{*}{0.3} & \multirow{2}{*}{0.01} & \multirow{2}{*}{0.003} & \multirow{2}{*}{0.1} & 1.000 & 1.1549 \\
\hline & & & & & & 100.000 & 1.4722 \\
\hline
\end{tabular}




\begin{tabular}{|c|c|c|c|c|c|c|c|}
\hline \multirow{2}{*}{ N..} & \multicolumn{5}{|c|}{ Parámetros } & \multirow{2}{*}{$\begin{array}{c}\text { Número } \\
\text { simulaciones }\end{array}$} & \multirow{2}{*}{$\begin{array}{l}\text { Vega generado } \\
\text { por el Método de } \\
\text { Trayectoria - Euler }\end{array}$} \\
\hline & $\mathbf{K}$ & $\square$ & $\kappa$ & $\theta$ & $\eta$ & & \\
\hline \multirow{2}{*}{6} & \multirow{2}{*}{105} & \multirow{2}{*}{0.3} & \multirow{2}{*}{0.03} & \multirow{2}{*}{0.003} & \multirow{2}{*}{0.1} & 1.000 & 6.9627 \\
\hline & & & & & & 100.000 & 7.2915 \\
\hline \multirow{2}{*}{7} & \multirow{2}{*}{105} & \multirow{2}{*}{0.3} & \multirow{2}{*}{0.003} & \multirow{2}{*}{0.003} & \multirow{2}{*}{0.1} & 1.000 & 7.0673 \\
\hline & & & & & & 100.000 & 7.4013 \\
\hline \multirow{2}{*}{8} & \multirow{2}{*}{105} & \multirow{2}{*}{0.3} & \multirow{2}{*}{0.01} & \multirow{2}{*}{0.005} & \multirow{2}{*}{0.1} & 1.000 & 7.0441 \\
\hline & & & & & & 100.000 & 7.3731 \\
\hline \multirow{2}{*}{9} & \multirow{2}{*}{105} & \multirow{2}{*}{0.3} & \multirow{2}{*}{0.01} & \multirow{2}{*}{0.001} & \multirow{2}{*}{0.1} & 1.000 & 7.0345 \\
\hline & & & & & & 100.000 & 7.3728 \\
\hline \multirow{2}{*}{10} & \multirow{2}{*}{105} & \multirow{2}{*}{0.3} & \multirow{2}{*}{0.01} & \multirow{2}{*}{0.003} & \multirow{2}{*}{0.2} & 1.000 & $14.0356-0.0067 \mathrm{i}$ \\
\hline & & & & & & 100.000 & $14.6048-0.0006 \mathrm{i}$ \\
\hline \multirow{2}{*}{11} & \multirow{2}{*}{105} & \multirow{2}{*}{0.3} & \multirow{2}{*}{0.01} & & & 1.000 & 3.5108 \\
\hline & & & & 0.003 & 0.05 & 100.000 & 3.6934 \\
\hline
\end{tabular}

